\title{
Erratum to: Observations of a Quasi-periodic, Fast-Propagating Magnetosonic Wave in Multiple Wavelengths and Its Interaction with Other Magnetic Structures
}

\author{
Y.-D. Shen · Y. Liu · J.-T. Su • H. Li • X.-F. Zhang • \\ Z.-J. Tian · R.-J. Zhao • A. Elmhamdi
}

Published online: 20 November 2013

(C) Springer Science+Business Media Dordrecht 2013

\section{Erratum to: Solar Phys (2013) 288:585-602 \\ DOI 10.1007/s11207-013-0395-4}

In the article, we ignored to acknowledge the support of the Visiting Professor Program at King Saud University, which provided the main financial support for our collaboration between the Yunnan Astronomical Observatory and the King Saud University, as well as this work. Therefore, the authors, especially Y. Liu and A. Elmhamdi, would like to acknowledge the support of the Visiting Professor Program at King Saud University. The authors apologize for this negligence.

The online version of the original article can be found under doi:10.1007/s11207-013-0395-4.

Y.-D. Shen $(\varangle) \cdot$ Y. Liu · X.-F. Zhang · Z.-J. Tian · R.-J. Zhao · A. Elmhamdi

Yunnan Astronomical Observatory, CAS, Kunming 650011, China

e-mail: ydshen@ynao.ac.cn

Y.-D. Shen · Y. Liu · J.-T. Su

Key Laboratory of Solar Activity, National Astronomical Observatories, CAS, Beijing 100012, China

Y.-D. Shen · H. Li

Key Laboratory of Dark Matter and Space Astronomy, Purple Mountain Observatory, CAS, Nanjing 210008, China

\section{A. Elmhamdi}

Physics and Astronomy Department, College of Science, King Saud University, P.O. Box 2455, Riyadh 11451, Saudi Arabia 